NEUROSCIENCE FOR NEUROLOGISTS

\title{
How to spot bias and other potential problems in randomised controlled trials
}

\author{
S C Lewis, C P Warlow
}

J Neurol Neurosurg Psychiatry 2004;75:181-187. doi: 10.1136/jnnp.2003.025833

T hese days, all clinical trials should be reported using the CONSORT guidelines ${ }^{1}$ (table 1); indeed JNNP recommends this in its instructions for authors. However, not all trials are reported in this way, and many journals do not insist on it. Thus some trials may have been carried out adequately but reported inadequately, while others have been carried out inadequately. Our aim in this article is to guide clinicians in what to look for in a report of a randomised controlled trial (RCT), so they can assess whether the trial was done adequately; we do not intend it to be a guide on how to do an RCT, as there are many such guides available. $^{2}$

The two crucial principles in clinical research are to minimise bias and to increase precision. If a study is not designed with these two principles in mind, no amount of analysis will sort them out. We will discuss some of the major biases to look out for, issues related to precision, and some other aspects of statistical analysis.

\section{BIAS}

Bias is any departure of results from the truth. An RCT is less susceptible to bias than other study designs for assessing therapeutic interventions. However, just because a study is randomised does not mean it is unbiased. There are at least seven important potential sources of bias in RCTs, which are discussed below. When assessing bias, it is important to consider its magnitude as well as its direction. Trials that have shown large treatment effects may still be positive after a small bias has been accounted for.

\section{Poor allocation concealment}

In a good trial, the treatment allocation is concealed during the randomisation procedure. In other words, at the time a clinician randomises a patient they will have no idea what the next treatment allocation is going to be. If allocation is concealed, it is not possible for a clinician to avoid a particular treatment allocation for a particular patient. For example, consider an RCT of surgical support stockings versus no treatment to prevent deep venous thrombosis after stroke. Say an incontinent patient arrives and the nurse is considering randomising. The nurse has access to the randomisation list and knows that the next random allocation is "stockings". Stockings on incontinent patients are a lot of work, as they need regular changing and washing, so the nurse chooses not to randomise the patient. Because incontinence is linked to stroke severity, in the long run this practice would cause the "stock- ings" arm of the trial to contain less severe strokes than the "no stockings" arm, which could bias the results of the trial, even though treatment allocation was randomised.

Good methods of allocation concealment include sequentially numbered, opaque, sealed envelopes; tamper-proof, sequentially numbered containers; pharmacy controlled lists; and telephone, fax, email, or internet contact with a central randomisation office. ${ }^{34}$

Imbalance in baseline prognostic variables In all trials, methods should be used to make sure that the treatment groups are as similar as possible. For example, there has been controversy over the results of the National Institute of Neurological Disorders and Stroke (NINDS) trial of thrombolysis for acute ischaemic stroke ${ }^{5}$ because the patients in the recombinant tissue plasminogen activator (rt-PA) group had less severe strokes than those in the control group, ${ }^{6}$ even though they were randomised. As less severe patients would be expected to have better outcomes than more severe patients, the trial results may have been biased in favour of rt-PA. The trialists adjusted for the imbalance in baseline severity in the analysis, but if the treatment groups had been comparable to start with, the arguments would not have arisen.

In a very large trial, randomisation should ensure that the treatment groups are balanced, but in small trials, imbalance can and does occur. Thus in smaller trials stratification is often used to increase the comparability of the treatment groups. Stratification ensures that roughly equal numbers of participants with a particular prognostic characteristic (perhaps age, or disease severity) will be allocated to each treatment group. It involves using separate randomisation lists for each prognostic subgroup (for example, for age $<80$ and age $\geqslant 80) .7$. It has been recommended ${ }^{8}$ that trials seeking to demonstrate the superiority of one treatment over another should consider stratifying randomisation when the overall sample size is small (for example, $<200$ patients per treatment arm for a dichotomous outcome), or when interim analyses are planned that will involve small sample sizes (stratification is recommended in all equivalence trials). The stratification factors must be strongly related to outcome. Thus in a trial of a treatment for acute stroke, one would stratify for stroke severity (which is strongly related to outcome), but not for sex (which is only weakly associated with outcome). In practice, it is probably more important that the reader can see that the treatment arms are balanced with respect to 


\begin{tabular}{|c|c|c|}
\hline \multicolumn{2}{|c|}{ Paper section and topic } & \multirow{2}{*}{$\begin{array}{l}\text { Description } \\
\text { How participants were allocated to interventions (for example, } \\
\text { "random allocation," "randomised," or "randomly } \\
\text { assigned"). }\end{array}$} \\
\hline $\begin{array}{l}\text { Title and } \\
\text { abstract }\end{array}$ & & \\
\hline \multirow{11}{*}{$\begin{array}{l}\text { Introduction } \\
\text { Methods }\end{array}$} & Background & Scientific background and explanation of rationale. \\
\hline & Participants & $\begin{array}{l}\text { Eligibility criteria for participants and the settings and locations } \\
\text { where the data were collected. }\end{array}$ \\
\hline & Interventions & $\begin{array}{l}\text { Precise details of the interventions intended for each group } \\
\text { and how and when they were actually administered. }\end{array}$ \\
\hline & Objectives & Specific objectives and hypotheses. \\
\hline & Outcomes & $\begin{array}{l}\text { Clearly defined primary and secondary outcome measures } \\
\text { and, when applicable, any methods used to enhance the } \\
\text { quality of measurements (for example, multiple observations, } \\
\text { training of assessors). }\end{array}$ \\
\hline & Sample size & $\begin{array}{l}\text { How sample size was determined and, when applicable, } \\
\text { explanation of any interim analyses and stopping rules. }\end{array}$ \\
\hline & $\begin{array}{l}\text { Randomisation - sequence } \\
\text { generation }\end{array}$ & $\begin{array}{l}\text { Method used to generate the random allocation sequence, } \\
\text { including details of any restriction (for example, blocking, } \\
\text { stratification). }\end{array}$ \\
\hline & $\begin{array}{l}\text { Randomisation - allocation } \\
\text { concealment }\end{array}$ & $\begin{array}{l}\text { Method used to implement the random allocation sequence. } \\
\text { (for example, numbered containers or central telephone), } \\
\text { clarifying whether the sequence was concealed until } \\
\text { interventions were assigned. }\end{array}$ \\
\hline & Randomisation - implementation & $\begin{array}{l}\text { Who generated the allocation sequence, who enrolled } \\
\text { participants, and who assigned participants to their groups. }\end{array}$ \\
\hline & Blinding (masking) & $\begin{array}{l}\text { Whether or not participants, those giving the interventions, } \\
\text { and those assessing the outcomes were blinded to group } \\
\text { assignment. When relevant, how the success of blinding was } \\
\text { evaluated. }\end{array}$ \\
\hline & Statistical methods & $\begin{array}{l}\text { Statistical methods used to compare groups for primary } \\
\text { outcome(s); methods for additional analyses, such as } \\
\text { subgroup analyses and adjusted analyses. }\end{array}$ \\
\hline \multirow[t]{6}{*}{ Results } & Participant flow & $\begin{array}{l}\text { Flow of participants through each stage (a diagram is strongly } \\
\text { recommended). Specifically, for each group report the } \\
\text { numbers of participants randomly assigned, receiving } \\
\text { intended treatment, completing the study protocol, and } \\
\text { analysed for the primary outcome. Describe protocol } \\
\text { deviations from study as planned, together with reasons. }\end{array}$ \\
\hline & $\begin{array}{l}\text { Recruitment } \\
\text { Baseline data }\end{array}$ & $\begin{array}{l}\text { Dates defining the periods of recruitment and follow up } \\
\text { Baseline demographic and clinical characteristics of each } \\
\text { group. }\end{array}$ \\
\hline & Numbers analysed & $\begin{array}{l}\text { Number of participants (denominator) in each group included } \\
\text { in each analysis and whether the analysis was by "intention to } \\
\text { treat" . State the results in absolute numbers when feasible (for } \\
\text { example, 10/20, not 50\%). }\end{array}$ \\
\hline & Outcomes and estimation & $\begin{array}{l}\text { For each primary and secondary outcome, a summary of } \\
\text { results for each group, and the estimated effect size and its } \\
\text { precision (for example, } 95 \% \text { confidence interval). }\end{array}$ \\
\hline & Ancillary analyses & $\begin{array}{l}\text { Address multiplicity by reporting any other analyses } \\
\text { undertaken, including subgroup analyses and adjusted } \\
\text { analyses, indicating those prespecified and those exploratory. }\end{array}$ \\
\hline & Adverse events & $\begin{array}{l}\text { All important adverse events or side effects in each } \\
\text { intervention group. }\end{array}$ \\
\hline \multirow[t]{2}{*}{ Discussion } & Interpretation & $\begin{array}{l}\text { Interpretation of the results, taking into account study } \\
\text { hypotheses, sources of potential bias or imprecision, and the } \\
\text { dangers associated with multiplicity of analyses and outcomes. }\end{array}$ \\
\hline & $\begin{array}{l}\text { Generalisability } \\
\text { Overall evidence }\end{array}$ & $\begin{array}{l}\text { Generalisability (external validity) of the trial findings. } \\
\text { General interpretation of the results in the context of current } \\
\text { evidence. }\end{array}$ \\
\hline
\end{tabular}

important prognostic baseline factors than to know the details of how this was achieved, although it is generally recommended that the stratification variables are presented in trial reports. ${ }^{1}$

\section{Unblinding and no blinding}

If anyone involved in a trial is aware of the allocated treatment, this may affect their judgement. In the Canadian cooperative trial of cyclophosphamide and plasma exchange in multiple sclerosis, neither of the active treatment groups was shown to be superior to placebo when the outcomes were blindly assessed by neurologists. ${ }^{9}$ However, in unblinded outcome assessment by neurologists there was an apparent treatment effect in one of the treatment groups. Trials can be designed so that the patient, the treatment team, the outcome assessor, and even the trial statistician and any data monitoring committee are all blinded to the allocated treatment. However, it may be impossible to blind the administering clinician to treatment allocation, particularly in trials of interventions such as surgery or physiotherapy. Blinding can even be difficult in some placebo controlled drug trials; for instance, intravenous rt-PA often causes bruising at the injection site. Probably the most important thing is for the person who assesses the primary outcome to be blinded to treatment allocation.

In general, the more blinding that is achieved, the less biased the trial results should be. It is worth noting that there is no single definition of the phrase "double blind", so trial 
reports should explain exactly who was blinded and how this was achieved. ${ }^{10}$

\section{Missing data}

In general, the more information from randomised patients that is missing, the more wary one should be of the trial results. For example, in a trial of a drug to prevent severe depression which actually works, more patients in the placebo group would become depressed. These would be more likely to stop taking a treatment that did not seem to work, and also to default from attending follow up appointments. If the results of those patients were missing from the final analysis, it would make the placebo group results look better than they actually were; at worst, it might then appear that the treatment was not working at all, or at best that it was working less well than was really the case. In the presence of much missing data, one never really knows what the true treatment effect is.

There are some circumstances when the exclusion of patients does not bias the results. ${ }^{11}$ For instance, it is allowable to exclude the data of a few ineligible patients who were mistakenly randomised into a trial because of human error. However, one must be sure that treatment is not potentially harmful for the ineligible patients, so it would not be appropriate to exclude patients with primary intracerebral haemorrhage who were inadvertently randomised into a trial of thrombolytic therapy. One must also be sure that the study results will not be applied to the ineligible patients. For instance, if an acute stroke treatment was to be given before computed tomography was done, then a few people with brain tumours would receive the treatment. Thus it would not be appropriate to exclude such patients from the analysis of a trial in which they had been inadvertently randomised.

Although excluding patients from the analysis in some circumstances does not bias the results, if many patients were excluded from a trial, one should question the quality of the trial design and execution. It should certainly be clearly described why any data are missing, and what effect this may have had on the results.

One way that trials can minimise the problem of missing patient information is to use central randomisation and follow up. For example, the FOOD trial ${ }^{12}$ is a family of three RCTs of feeding after stroke that uses this system. Baseline data are collected during a randomisation phone call before randomisation actually happens, and so are $100 \%$ complete. The central office follows up all randomised patients, and in February 2001, of 3012 patients randomised, only 10 had permanently missing primary outcome data.

\section{Lack of intention to treat analysis}

Intention to treat means that patients are analysed in the treatment group they were randomised to, whatever happens later. Some trials analyse the data using an on-treatment analysis where patients are only analysed if they received the treatment they were randomised to. An intention to treat analysis preserves the randomisation process. It has the advantage of being more like standard clinical practice (where patients will start on other treatments if the first treatment they are given does not agree with them, or they may choose not to take any treatment at all). It also takes care of unexpected adverse events (patients cannot "drop out" of the trial analysis if they have an adverse effect of treatment) and is less open to fraud (the trialists cannot exclude any patients who did not achieve the hoped-for outcome).

Figure 1 shows an example of how an on-treatment analysis can cause bias in practice. Patients with carotid transient ischaemic attacks are randomised to receive either carotid surgery or no surgery. If there is a delay of a few days between randomisation and surgery, patients may have a primary outcome event (in this case a stroke) after randomisation but before the surgery is done. In the surgery group, patients who suffer a severe stroke or die will not receive surgery (and would therefore be removed from the on-treatment analysis). However, in the no surgery group, if patients have a stroke or die within a few days of randomisation, they will have received their allocated treatment (no surgery) and would therefore be counted in the on-treatment analysis. The omission of early strokes from the surgery group would cause fewer strokes to be counted in the surgery arm, and therefore the on-treatment analysis would be biased in favour of surgery. The intention to treat analysis includes all patients and is therefore unbiased.

\section{Counting death as a good outcome}

It is important when reading a trial report to consider how death has been analysed and what effect this may have had on the results. For instance, the trial may measure the proportion of patients who were disabled at follow up, using all patients randomised as the denominator. In this case, the trial is really comparing the proportion of patients who were alive and disabled at follow up to the proportion of patients who were not disabled at follow up, and this latter group includes both those who were alive and not disabled, and those who were dead. Thus death has been included as a good outcome. It would be more sensible to measure the proportion of patients who were alive and not disabled at follow up. ${ }^{13}$

\section{Competing interests}

It has been shown that research funded by pharmaceutical companies is more likely to have outcomes favouring the sponsor than research funded from other sources. ${ }^{14} 15$ Pharmaceutical company research is certainly not of poorer quality than other research, but the companies may have a tendency not to publish unfavourable results. The number of industry sponsored trials in stroke is increasing, ${ }^{16}$ so this problem is not going to go away, but there are now guidelines on the relation between sponsors and investigators that may improve the situation. ${ }^{17}$

\section{PRECISION}

A treatment effect estimate is precise when the confidence interval around it is very tight, and we are therefore confident about its magnitude.

\section{Size of trials}

Larger trials provide more precise estimates of treatment effects than small trials, and they may allow a few sensible and predefined subgroup analyses ${ }^{18}$. Small trials, with wide confidence intervals around their estimates of treatment effect, are clinically uninformative (although they may add to an existing meta-analysis or generate enthusiasm to do further bigger trials).

Trials that use continuous outcome measures (for example, blood pressure or time taken to walk 10 metres) generally have greater precision than trials of the same size that use binary outcome measures (for example, dead versus alive, hypertensive versus not). However, with continuous outcome measures there may be more of a problem with missing data, and it may be unclear how to score dead patients.

RCTs that measure the outcomes that really matter to patients (such as death or dependency) often require large numbers of patients to be followed up for long periods. ${ }^{19}{ }^{20}$ To reduce the size of the trial, some trialists use surrogate outcomes. For instance, to investigate whether a neuroprotective drug reduces death and dependency after stroke, one would probably have to randomise thousands of patients, which would take several years. If one used the size of the 


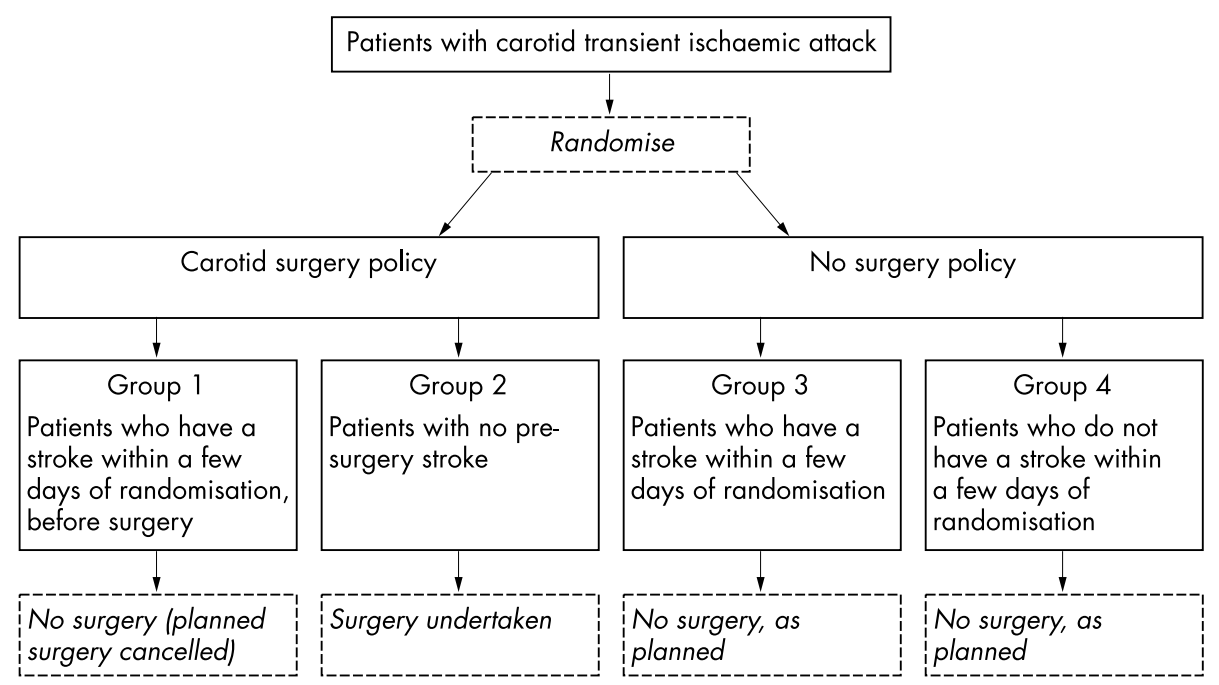

Figure 1 An example from a carotid surgery trial showing how an intention to treat analysis is less biased than an on-treatment analysis. An ontreatment analysis compares group 2 with groups 3 and 4 . The omission of group 1 will cause fewer strokes to be counted in the surgery arm, and therefore this analysis will be biased in favour of surgery. An intention to treat analysis compares groups 1 and 2 with groups 3 and 4 , thus including all strokes.

infarct on magnetic resonance imaging 48 hours posttreatment as a surrogate marker for efficacy, one could substantially reduce the size and duration of the trial. This would mean that a new treatment could be proved efficacious and licensed much earlier, and so benefit many more patients. However, such surrogate outcomes often do not prove to be effective substitutes for the true clinical outcome. ${ }^{19}$ One of the reasons for this failure may be that the surrogate marker and the clinical outcome are on different causal pathways. Alternatively, several independent processes may cause the disease, only one of which involves the surrogate marker. ${ }^{20}$ For instance, the early "inflammatory" stage of multiple sclerosis may be detected on magnetic resonance imaging, but this does not necessarily relate to progression of the disease or later disability. Thus if a trial used this evidence from MRI as a surrogate marker of clinical outcome in multiple sclerosis, it would only show the effects of treatment on the early inflammatory lesions.

\section{Power versus confidence intervals}

The concept of power is very important when designing a study. Assume that a drug reduces the absolute risk of having a stroke by $10 \%$. If a trial to measure this treatment effect was repeated over and over, it would sometimes estimate it to be greater than $10 \%$ and sometimes less than $10 \%$. In some of the trials, the estimated treatment effect would be so small that the result would be statistically non-significant (as the confidence interval for the estimate of the treatment effect would overlap "no effect"). If the trial is designed to have $80 \%$ power, then, if the treatment effect truly exists, if the trial was repeated 100 times a statistically significant treatment effect would be found in 80 of them. So one in every five trials would falsely show a non-statistically significant result.

However, although this is an essential concept when designing a trial, once a trial has been completed it is more important to concentrate on the width of the confidence interval around the treatment effect than on the power itself. $^{21}$ When the study was designed, the power calculation was based on a guess at what the treatment effect might be. After the study is completed, the result is known and it makes no sense to use prestudy guesses to interpret the result. The confidence interval around the treatment effect is based on the actual trial result, and this is what really counts.

\section{Early stopping}

Some trials are planned to be large, but they end up small because they are stopped early owing to an apparently huge beneficial effect or a harmful effect. The results of such trials should be treated with some scepticism, because if they had been allowed to continue, the final estimated treatment effect may well have been much smaller. ${ }^{22}$ In the initial stages of a trial, the treatment effect tends to zig and zag through some quite extreme values before settling down, and thus trials that stop early may just have stopped on one of the random highs or lows in the treatment effect estimate. Of course, there may be ethical reasons why a trial has to stop because of early unexpected harmful effects. However, for some treatments (such as thrombolysis for acute stroke) and for many surgical interventions, the trial data monitoring committee should have carefully considered the possibility that any early harm may be outweighed by later benefit.

\section{"Absence of evidence" and "evidence of absence"}

Care should be taken in the interpretation of non-statistically significant results. It is quite common for investigators to confuse "absence of evidence of effectiveness" with "evidence of absence of effectiveness." Take, for example, a trial examining the effect of a drug on death or dependency after stroke. Nine of 20 patients treated with the drug are dead or dependent at follow up compared with 10 of 20 untreated patients, giving a $\mathrm{p}$ value of 0.8 . However, although the point estimate for the absolute treatment effect is 5\% (10/20 minus $9 / 20$ ), the $95 \%$ confidence interval for the difference in proportions runs from $-24 \%$ to $+33 \%$. It is therefore quite plausible that the treatment could cause great harm, or great benefit. Thus the conclusion of this trial is that we do not know whether the drug works, and we would need to do a larger trial to find out.

To assess "evidence of absence of effectiveness," a trial needs to be designed as an equivalence trial. RCTs cannot prove that two treatments are of identical efficacy, but they can prove that two treatments are of similar efficacy. ${ }^{23}$ In equivalence trials, trialists need to prespecify what they mean by clinical equivalence. Usually a range of equivalence for the treatment difference is defined such that any value within 
the range is deemed clinically unimportant. ${ }^{24}$ When the trial results are published, to show that two treatments are equivalent the confidence interval for the treatment effect must fall wholly within this predefined range of equivalence. ${ }^{25}$

\section{ANALYSIS AND INFERENCES Baseline differences}

In RCTs, many consider that it is not appropriate to test for differences in the level of baseline factors between treatment groups. ${ }^{26}{ }^{27}$ In an RCT with two treatment groups, such tests are testing the hypothesis that the two groups come from the same population. However, if the randomisation was fair, then the two groups will certainly have come from the same population, and one ends up testing whether the randomisation was fair, not whether the two groups had similar characteristics. ${ }^{26}$ One should be very wary if no baseline data are presented at all.

\section{Adjusted versus unadjusted analyses}

Results of RCTs can be presented either adjusted or unadjusted for any differences in baseline prognostic variables. Differences between treatment groups may bias the results (as explained earlier), and "adjustment" is any statistical method that alleviates this problem. To understand adjustment, consider the following example. Patients who suffer strokes of moderate severity generally have worse outcomes than those of mild severity. In a trial of a drug in acute stroke, let us assume the proportion of patients with mild stroke was much higher in the treated group than in the untreated group. An unadjusted analysis would overestimate the treatment effect, as the treated patients were more likely to do well before treatment than the untreated patients. However, one can calculate the treatment effect in just the mild patients, and in just the moderate patients, and then average these two (table 2). The statistical procedures used to adjust results are often more complex than this, but they follow the same basic principle.

When reading a trial report, the unadjusted analyses are easier to understand, as sometimes the adjusted results seem to have come out of a statistical "black box" and it is unclear exactly what has been done. However, adjusted analyses have statistical advantages in some cases. The key issue is the correlation between each baseline variable and outcome. ${ }^{28}$ If this is high (say $>0.5$ ), then adjusting the analyses for the baseline variable is important. This might happen, for instance, if the same variable is measured at baseline and as an outcome after treatment (for example, measuring blood pressure before and after treatment in a trial of a blood pressure lowering drug). However, if the correlation between a baseline variable and outcome is low, then there is probably no point adjusting for it. These arguments apply no matter how large the trial is, and whether or not there has been stratification for the baseline variable in the randomisation process. When reading a trial report, one should be most convinced when both adjusted and unadjusted analyses are presented and agree with one another.

\section{Subgroups and multiple testing}

A report of an RCT will often contain at least one subgroup analysis, such as the treatment effect in young versus old patients. Although there are many guidelines on appropriate ways to do such analyses, inappropriate analyses are still frequently presented. ${ }^{29}$

In fig 2, the effect of aspirin in male and female subjects from the Canadian cooperative study group ${ }^{30}$ is shown-a landmark trial in its time. The results in fig 2 are shown in a forest plot, ${ }^{31}$ which is a convenient way of presenting subgroup analysis results. In male subjects, the effect of aspirin was statistically significant, and in female subjects it was not. However, this method of inspection of subgroup $p$ values is incorrect. If one group has more subjects (as in this case, where there are twice as many men in the trial as women), then it is far more likely to have a significant $p$ value, and this has nothing to do with the magnitude of the treatment effect in that group. The correct way to do a subgroup analysis is to compare the size of the treatment effect in one subgroup with the size of the treatment effect in the other. ${ }^{32}$ In this case, the correct $p$ value is 0.008 , which is statistically significant. However, this really shows the dangers of subgroup analysis, because this one was not prespecified, was not biologically plausible, and was later proved wrong by the antiplatelet trialists' collaboration metaanalysis. $^{33}$

If one examines the effects of treatment in 20 subgroups in an RCT, one will obtain, on average, one spurious false positive result at $\mathrm{p}=0.05$ just by chance. This is more likely than the chance of rolling two sixes with a pair of dice, which happens with a probability of 0.028 . Sometimes spurious subgroup results can be dramatic. ${ }^{34}$ If inappropriate subgroup analyses have been done (such as inspection of subgroup p values), the number of false positive results could increase drastically. ${ }^{29}$ If many subgroup analyses are shown, it is quite likely that a couple of "statistically significant" subgroup effects will occur, but this does not mean that they are clinically meaningful. The ISIS 2 trial $^{35}$ showed that aspirin was less beneficial to people born under the star sign of Gemini or Libra than other star signs, which puts the results of the other subgroup analyses into perspective. If only a few subgroup analyses are shown (possibly spread over several publications from the same trial) but they are all statistically significant, one should be very cautious about their interpretation, because the investigator may well have only presented the statistically significant results, and may have hidden the hundreds of non-significant results.

Table 2 Adjusted and unadjusted analyses - a simple example from a hypothetical trial of a treatment for acute stroke where, for some reason, a greater number of mild patients was randomised to the treatment being tested

\begin{tabular}{|c|c|c|c|c|c|c|c|c|c|}
\hline & \multicolumn{3}{|c|}{ All patients } & \multicolumn{3}{|c|}{ Patients with moderate stroke } & \multicolumn{3}{|c|}{ Patients with mild stroke } \\
\hline & Treated & Untreated & Total & Treated & Untreated & Total & Treated & Untreated & Total \\
\hline Dead or dependent & 250 & 420 & 670 & 100 & 360 & 460 & 150 & 60 & 210 \\
\hline Alive and independent & 750 & 580 & 1330 & 150 & 390 & 540 & 600 & 190 & 790 \\
\hline Total & 1000 & 1000 & 2000 & 250 & 750 & 1000 & 750 & 250 & 1000 \\
\hline
\end{tabular}

Calculating relative risks of being dead and dependent:

Overall unadjusted treatment effect is $250 / 1000 \div 420 / 1000=0.60$

Treatment effect for those with moderate stroke is $100 / 250 \div 360 / 750=0.83$

Treatment effect for those with mild stroke is $150 / 750 \div 60 / 250=0.83$

So an overall adjusted treatment effect would be 0.83 . 


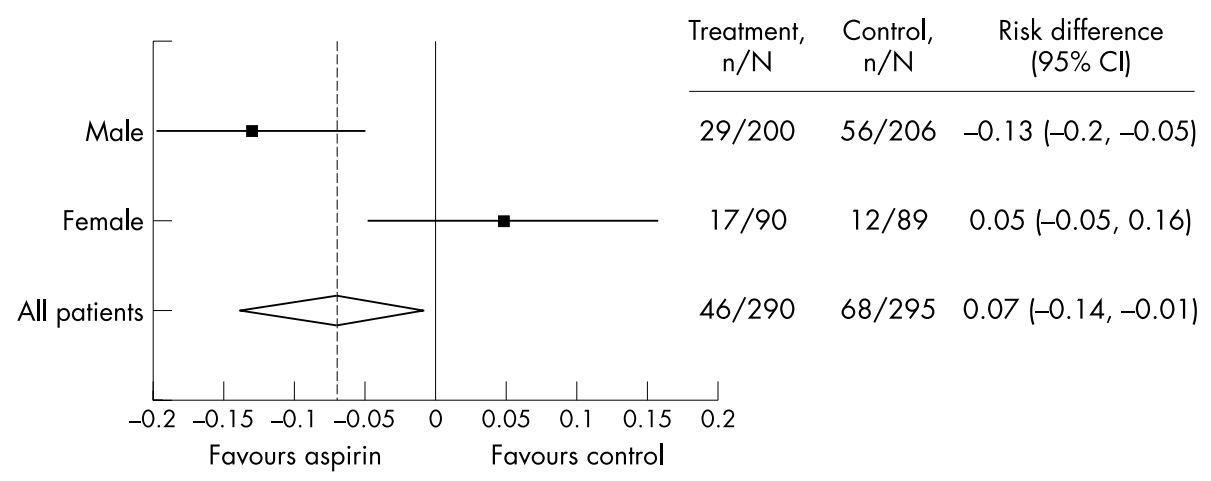

Figure 2 The dangers of subgroup analysis: the Canadian cooperative study group 1978-difference in risk of stroke or death in 585 patients with transient ischaemic attacks or stroke treated long term with aspirin versus no aspirin. $n=$ number of patients with stroke or death; $N=$ total number of patients randomised. $\mathrm{Cl}$, confidence interval.

The results of subgroup analyses are more believable if there are only a few subgroups, predefined using biologically plausible arguments or from an a priori hypothesis generated from previous studies, from a trial large enough to stand a good chance of finding statistically significant treatmentsubgroup interactions, ${ }^{27}$ and best of all, confirmed in a completely separate RCT. But in general, one should put most emphasis on the primary result in all patients.

The effect of false positive results from multiple testing occurs for all analyses, not just subgroup analyses. If the investigator has analysed 20 different outcomes, it is likely that one will be statistically significant just by chance.

Sometimes, a trial measures outcome at several time points, and undertakes a separate analysis at each one. Again, it is likely that one will be significant just by chance. Matthews et al describe appropriate methodology for such situations. $^{36}$

\section{Unit of analysis}

Most of the examples discussed so far have related to standard two arm parallel group trials - that is, patients are randomised to one treatment or another, and after a period of time an outcome is measured on each patient. However, there are many other types of trial, and when reading a trial report, it is important to check whether the analysis is appropriate to the design, or whether the design is appropriate at all. For instance, crossover trials are used in some areas of medicine. In these trials each patient receives both treatments, but the order in which they receive them is randomised. Crossover trials have the advantage that each patient acts as their own control. However, they only work in chronic conditions, where the treatment cannot cure the disease, only alleviate the symptoms. Thus they might be suitable in migraine, but not in meningitis.

In most trials, the unit of randomisation and the unit of analysis are the same. Patients are randomised to treatments, and then the outcome is measured and analysed at the level of the patient-that is, the outcome measure is measured once on each patient (for example, death). However, there are trials where the unit of randomisation is not the same as the unit of analysis. In a trial of a drug to reduce the size of secondary brain tumours, the patient would be randomised, but if patients had more than one tumour, the outcome might be recorded at the level of the tumour. The report of such a trial should describe clearly that the analysis has taken this design into account. It is important that this is done, as one would expect the tumours in one patient to behave quite similarly, thus reducing the variability in the estimate of treatment effect. Similar problems occur in cluster randomised trials. These are trials where the unit of randomisation is, for instance, a general practice surgery, or a residential home, but where the outcome is measured at the level of the patient. This design must be taken into account in the analysis. ${ }^{37}$

\section{Recurrent events}

In some areas of medicine, outcomes tend to be measured in terms of rates of events. For instance, in multiple sclerosis one may be interested in the number of relapses that patients have within a fixed period. Similar data are obtained from trials examining numbers of epileptic fits or headaches. These data need to be analysed using appropriate statistical methods which take account of the fact that some patients are much more likely to suffer recurrent events than others. ${ }^{38}$ One should think carefully about what such results actually mean. In epilepsy, one of 10 patients having 100 fits is rather different from 10 of 10 patients having 10 fits each. Although the mean number of fits is 10 in both scenarios, they are clearly quite different situations.

\section{Adverse events}

In addition to considering the efficacy of a treatment, it is essential to consider safety..$^{39}$ In the elderly, there can be more adverse effects of treatments than in the young (such as postural hypotension and fainting in blood pressure lowering trials). Even minor adverse events can be important when considered across large populations. To take an extreme example, let us assume that it was decided to give the whole population over the age of 50 a statin to lower their cholesterol levels, but that in many people this caused them to have mild numbness in the hands. Such an adverse effect might not be seen as a problem in a person who was seriously ill and very likely to suffer a heart attack in the coming year unless treated. However, in previously healthy people, the adverse effect would not be accepted, and across a whole population it could be disastrous. One should be wary of trials where no adverse effects of treatment are reported, as there are no magic bullets in medicine. One should also be wary of trials where the length of follow up has been very short, as adverse events may only arise after a longer period. Few RCTs will be big enough to detect rare adverse events, but small trials will not even be able to detect common adverse events. In order to detect adverse effects reliably, effective postmarketing surveillance in large numbers of patients, or a case-control study, is required.

The assessment of adverse effects is particularly important in equivalence trials. It is not enough to prove that two treatments are equivalent in terms of efficacy. A new treatment must be as safe as, or safer than, the old one (and if it is not significantly safer than the old treatment then it should be shown to be cheaper or more convenient). 


\section{Generalisability and interpretation}

The result of an RCT is only applicable to day to day practice if the patients included in the trial were similar to those who would be treated in practice. Thus if a treatment has only been tested in men aged under 65 , it is generally impossible to know whether it will benefit a 95 year old woman. On balance, trials with broad inclusion criteria are more generalisable than those with strict criteria. By examining the inclusion and exclusion criteria, and the baseline data, clinicians should consider whether the trial sample is reasonably representative of the people they wish to treat. ${ }^{40}$ However, more emphasis should be placed on the overall outcome of the trial than on the results for one particular subgroup within the trial. ${ }^{41}$

\section{THE FUTURE}

Although in the past the quality of trial reporting has been poor, it does appear to be improving. ${ }^{16}{ }^{42}$ The use of the CONSORT guidelines ${ }^{1}$ by more journals will increase quality further. In the future, let us hope we can be more able to believe what we read.

\section{CONCLUSIONS}

Allocation should be concealed during randomisation. Outcomes should be defined carefully. Outcome assessors should be blind to treatment allocation. It should be clear why missing data are missing. Analyses should be on the basis of intention to treat. It is worth bearing in mind that just because a trial says it is "intention to treat" and that allocation is concealed, it does not mean that these statements are true. It is important to read a trial report carefully to make sure that the authors knew the precise definitions of these terms.

One should be particularly cautious not to overinterpret the results of subgroup analyses. We all want to find treatments that work, and we can get very excited when we think we have found something, but we need to remember that some things are just too good to be true.

\section{ACKNOWLEDGEMENTS}

We would like to thank Jon Stone and Rustam Al Shahi for helpful comments on an earlier draft.

\section{Authors' affiliations}

S C Lewis, Neurosciences Trials Unit, Division of Clinical Neurosciences, Western General Hospital, Edinburgh, UK

C P Warlow, Division of Clinical Neurosciences, University of Edinburgh

Competing interests: none declared

Website of interest: CONSORT: http://www.consort-statement.org/

\section{REFERENCES}

1 Moher D, Schulz KF, Altman D. The CONSORT statement: revised recommendations for improving the quality of parallel-group randomized trials. JAMA 2001;285:1987-91.

2 Pocock SJ. Clinical trials - a practical approach. Chichester: John Wiley, 1983.

3 Altman DG, Schulz KF. Concealing treatment allocation in randomised trials. BMJ 2001;323:446-7.

4 Schulz KF, Grimes DA. Allocation concealment in randomised trials: defending against deciphering. Lancet 2002;359:614-18.

5 National Institute of Neurological Disorders and Stroke rt-PA stroke study group. Tissue plasminogen activator for acute ischemic stroke. N Engl J Med 1995;333:1581-7.

6 Mann J. Truths about the NINDS study: setting the record straight. West J Med 2002;176:192-4.

7 Roberts C, Torgerson D. Randomisation methods in controlled trials. BMJ 1998;317:1301.
8 Kernan WN, Viscoli CM, Makuch RW, et al. Stratified randomisation for clinical trials. J Clin Epidemiol 1999;52:19-26.

9 Noseworthy JH, Ebers GC, Vandervoort MK, et al. The impact of blinding on the results of a randomised, placebo-controlled multiple sclerosis clinical trial. Neurology 1994:44:16-20.

10 Schulz KF, Chalmers I, Altman DG. The landscape and lexicon of blinding in randomized trials. Ann Intern Med 2002;136:254-9.

11 Fergusson D, Aaron SD, Guyatt G, et al. Post-randomisation exclusions: the intention to treat principle and excluding patients from analysis. $B M$ 2002;325:652-4

12 The FOOD trial collaboration. Performance of a statistical model to predict stroke outcome in the context of a large, simple, randomized, controlled trial of feeding. Stroke 2003;34:127-33.

13 Lubsen J, Kirwan BA. Combined endpoints: can we use them? Stat Med 2002;21:2959-70.

14 Bekelman JE, Li Y, Gross CP. Scope and impact of financial conflicts of interest in biomedical research - a systematic review. JAMA 2003;289:454-65.

15 Lexchin J, Bero LA, Djulbegovic B, et al. Pharmaceutical industry sponsorship and research outcome and quality: systematic review. BMJ 2003;326: $1167-70$.

16 Kidwell CS, Liebeskind DS, Starkman S, et al. Trends in acute ischaemic stroke trials through the 20th century. Stroke 2001;32:1349-59.

17 Donnan GA, Davis SM, Kaste M, for the international trial subcommittee of the international stroke liaison committee, American Stroke Association. Recommendations for the relationship between sponsors and investigators in the design and conduct of clinical stroke trials. Stroke 2003;34:1041-5.

18 Warlow C. Advanced issues in the design and conduct of randomized clinical trials: the bigger the better? Stat Med 2002;21:2797-805.

19 Fleming TR, DeMets DL. Surrogate endpoints in clinical trials: are we being misled? Ann Intern Med 1996;125:605-13.

20 Bucher HC, Guyatt GH, Cook DJ, et al. For the evidence-based medicine working group. Users' guides to the medical literature, XIX. Applying clinical trial results. A. How to use an article measuring the effect of an intervention on surrogate end points. JAMA 1999;282:771-8.

21 Goodman SN, Berlin JA. The use of predicted confidence intervals when planning experiments and the misuse of power when interpreting results. Ann Intern Med 1994;121:200-6.

22 Wheatley K, Clayton D. Be skeptical about unexpected large apparent treatment effects: the case of an MRC AML1 2 randomization. Control Clin Trials 2003;24:66-70.

23 Siegel JP. Equivalence and noninferiority trials. Am Heart J 2000; 139:S160-70.

24 Ebbutt AF, Frith L. Practical issues in equivalence trials. Stat Med 1998;17:1691-701.

25 Jones B, Jarvis $\mathrm{P}$, Lewis JA, et al. Trials to assess equivalence: the importance of rigorous methods. BMJ 1996;313:36-9.

26 Altman DG, Doré CJ. Randomisation and baseline comparisons in clinical trials. Lancet 1990;335:149-53.

27 Assmann SF, Pocock SJ, Enos LE, et al. Subgroup analysis and other (mis)uses of baseline data in clinical trials. Lancet 2000;355:1064-9.

28 Pocock SJ, Assmann SE, Enos LE, et al. Subgroup analysis, covariate adjustment and baseline comparisons in clinical trial reporting: current practice and problems. Stat Med 2002;21:2917-30.

29 Brookes ST, Whitley E, Peters TJ, et al. Subgroup analyses in randomised controlled trials: quantifying the risks of false-positives and false-negatives. Health Technol Assess 2001;5:33.

30 The Canadian Cooperative Study Group. A randomized trial of aspirin and sulfinpyrazone in threatened stroke. N Engl J Med 1978;299:53-9.

31 Lewis S, Clarke M. Forest plots: trying to see the wood and the trees. BMJ 2001;322:1479-80.

32 Matthews JNS, Altman DG. Statistics notes: Interaction 3: how to examine heterogeneity. BMJ 1996;313:862.

33 Antiplatelet Trialists' Collaboration. Collaborative overview of randomised trials of antiplatelet therapy. I: Prevention of death, myocardial infarction, and stroke by prolonged antiplatelet therapy in various categories of patients. BMJ stroke by prolonged
1994;308:81-106.

34 Clarke M, Halsey J. DICE 2: a further investigation of the effects of chance in life, death and subgroup analyses. Int J Clin Pract 2001;55:240-2.

35 ISIS-2 collaborative group. Randomised trial of intravenous streptokinase, oral aspirin, both, or neither among 17187 cases of suspected acute myocardial infarctio: ISIS-2. Lancet 1988;ii:349-60.

36 Matthews JNS, Altman DG, Campbell MJ, et al. Analysis of serial measurements in medical research. BMJ 1990;300:230-5.

37 Kerry SM, Bland JM. Trials which randomize practices I: how should they be analysed? Fam Pract 1998;15:80-3

38 Glynn RJ, Buring JE. Ways of measuring rates of recurrent events. BMJ 1996;312:364-7.

39 Cuervo LG, Clarke M. Balancing benefits and harms in health care - we need to get better evidence about harms. BMJ 2003;327:65-6.

40 Schulz KF, Grimes DA. Sample size slippages in randomised trials: exclusions and the lost and wayward. Lancet 2002;359:781-5.

41 Collins R, MacMahon S. Reliable assessment of the effects of treatment on mortality and major morbidity, I: clinical trials. Lancet 2001;357:373-80.

42 Bath F, Owen V, Bath P. Quality of full and final publications reporting acute stroke trials. A systematic review. Stroke 1998;29:2203-10. 